\title{
ELABORACIÓN DE UNA MERMELADA A PARTIR DEL PECIOLO DE RUIBARBO (Rheum Rhabarbarum)
}

\section{ELABORATION OF A JAM FROM THE PETIOLES OF RHUBARB (Rheum Rhabarbarum)}

\section{Granados C. Clemente ${ }^{1^{\star}}$, Torrenegra A. Miladys E. ${ }^{1,2}$, Díaz M. Olinda ${ }^{3}$.}

\footnotetext{
${ }^{1}$ Facultad de Ingeniería, Universidad de Cartagena. Grupo de Investigación en Ingeniería, Innovación, Calidad Alimentaria y SaludINCAS Ingeniero de Alimentos. Magister en Ciencias y Tecnología de Alimentos. Profesor Titular. *Correo electrónico: clementecondeg@gmail.com. Teléfono: (575)6699771 Celular: (57)3158332900. Dirección: Universidad de Cartagena, Facultad de Ingenierías, Campus de la Piedra de Bolívar, Cartagena, Colombia.

${ }^{2}$ Centro de Comercio y Servicios, Regional Bolívar (SENA). Ingeniera de Alimentos. Magister en Ciencias y Tecnología de Alimentos. Grupo de Investigación Biotecnología e Innovación (GIBEI). Cartagena, Colombia.

${ }^{3}$ Facultad de Ingeniería, Universidad de Cartagena. Grupo de Investigación en Ingeniería, Innovación, Calidad Alimentaria y SaludINCAS Ingeniero de Alimentos. Cartagena, Colombia.
}

Recibido 21 de Julio 2016; aceptado 30 de octubre de 2016

\section{RESUMEN}

El ruibarbo (Rheum Rhabarbarum) es una especie de planta fanerógama perteneciente a la familia Polygonaceae, de gran aceptación en los trópicos, donde se consume fresca y procesada. El objetivo del trabajo fue el de elaborar una mermelada a partir del peciolo de ruibarbo ( $R$. Rhabarbarum). Se trabajaron 5 formulaciones de mermeladas a partir del peciolo, empleando azúcar blanco y panela como endulzante. Se evaluaron las características fisicoquímicas y 
microbiológicas de los productos obtenidos. Todas las formulaciones presentaron valores aceptados por la Norma Técnica Colombiana (NTC 285), evidenciando en las formulaciones con presencia de panela un aumento en el contenido de calcio, hierro, fibra total y humedad.

Palabras clave: Ruibarbo, peciolo, mermelada, azúcar, panela.

\section{ABSTRACT}

Rhubarb (Rheum Rhabarbarum) is a species of seagrass plant belonging to the family Polygonaceae, of great acceptance in the tropics, where it is consumed fresh and processed. The objective of the work was to make a marmalade from the petiole of rhubarb (R. Rhabarbarum). Five formulations of jams were worked from the petiole, using white sugar and brown sugar as a sweetener. The physico-chemical and microbiological characteristics of products obtained were evaluated. All the formulations presented values accepted by the Colombian Technical Standard (NTC 285), demonstrating in the formulations with presence of brown sugar. an increase in the content of calcium, iron, total fiber and humidity.

Keywords: Rhubarb, petiole, jam, sugar and brown sugar.

\section{INTRODUCCIÓN}

\section{El Ruibarbo (Rheum Rhabarbarum) antioxidantes promisorios (Huber, T., et al., pertenece a la familia poligonácea que 2009). Siendo el peciolo la parte comestible contiene derivados del metilantraceno, de la planta (McDougall, G., et al., 2010), son quinonas (Öztürk, M., et al., 2007), y redondeados en su parte dorsal, y compuestos fenólicos que han sido aplastados verticalmente de color rojo o reportados como antibacterianos y}


@. LIMENTECH CIENCIA Y TECNOLOGÍA ALIMENTARIA ISSN 1692-7125. Volumen 14, No. 2, p. 33- 41, año 2016 Facultad de Ingenierías y Arquitectura Universidad de Pamplona

verde dependiendo de la variedad (Paula, J., y Martins, A., 2000).

El procesamiento tecnológico del ruibarbo ofrece opciones de conservación del peciolo fresco para extender su vida útil. El peciolo contiene polifenoles, los cuales son metabolitos secundarios de las plantas con actividad antioxidante beneficiosa para la salud humana (Hisashi, M., et al., 2001). En la Tabla de Composición de Alimentos de la USDA, 2016 (United States Department of Agriculture), se reportan valores de calorías, humedad, grasas, carbohidratos, fibras, cenizas, calcio, fósforo, hierro, manganeso, magnesio, zinc, sodio, potasio, riboflavina, tiamina, niacina, ácido fólico, ácido pantoténico, vitamina B6, y ácido ascórbico, para el ruibarbo crudo. Sin embargo, en esta tabla no se incluyen los polifenoles.

Dentro de los métodos de procesamiento de alimentos, la concentración implica evaporación del agua y una disminución de su actividad acuosa; como es el caso del proceso tecnológico de la elaboración de mermeladas (Marquina, V., et al., 2008), en donde participan además otros adjuntos a la conservación de la misma: el uso de conservadores, la acidez alta de la formulación, la alta concentración de azúcar y los tratamientos térmicos (López, M., et al., 2011; Torrenegra, M., et al., 2015). objetivo del presente trabajo consistió en elaborar una mermelada a partir del peciolo de ruibarbo ( $R$. Rhabarbarum).

\section{MATERIALES Y MÉTODOS}

\section{Recolección del material vegetal}

Con base en los registros de las colecciones del Herbario Regional Catatumbo-Sarare (HECASE) de la Universidad de Pamplona, los peciolos de las plantas de ruibarbo (Rheum rhabarbarum) fueron recolectados en una vereda del municipio de Pamplona Norte de Santander $\left(7^{\circ} 22^{\prime} 34^{\prime \prime N} 72^{\circ} 38^{\prime} 54^{\prime \prime O}\right)$. Se recolectaron $5 \mathrm{Kg}$ de material bajo la supervisión del Magíster en Sistemática Vegetal, Luis Roberto Sánchez Montaño quien realizó la identificación taxonómica de la especie (Yáñez, C. y Santafé, G., 2012).

\section{Caracterización fisicoquímica del peciolo} y sus derivados.

Los contenidos de fibra cruda, cenizas, grasa, carbohidratos, proteína y minerales se determinaron según la metodología descrita Morilla y Delgado (2012). El color se determinó utilizando un espectrofotómetro de esfera, modelo SP64, X-RITE Inc, MI, 
(a)LIMENTECH CIENCIA Y TECNOLOGÍA ALIMENTARIA ISSN 1692-7125. Volumen 14, No. 2, p. 33- 41, año 2016 Facultad de Ingenierías y Arquitectura Universidad de Pamplona

USA), con iluminante D65 y un observador de $10^{\circ}$ como referencia. A partir de los espectros de reflexión se obtuvieron las coordenadas de color CIE-La*b*, donde $L^{*}$ es la luminosidad, $a^{*}$ es la cromaticidad verde (-) a rojo (+) y $b^{*}$ es la cromaticidad azul (-) a amarillo (+) (Santamaría, F., et al., 2010).

\section{Preparación de las mermeladas.}

Las formulaciones de las mermeladas de ruibarbo se diseñaron teniendo en cuenta los promedios de los \% mínimos de sólidos solubles (SS), utilizando diferentes concentraciones de azúcar y panela. (Tabla 1).

Tabla 1. Formulaciones de las mermeladas de ruibarbo (Rheum Rhabarbarum)

\begin{tabular}{|c|c|c|c|c|c|}
\hline $\begin{array}{c}\text { Ingredie } \\
\text { ntes }\end{array}$ & $\begin{array}{c}\mathbf{1 0 0} \\
\mathbf{A}\end{array}$ & $\begin{array}{c}\mathbf{7 5 : 2} \\
\mathbf{5} \\
\mathbf{A} / \mathbf{P}\end{array}$ & $\begin{array}{c}50: 5 \\
\mathbf{0} \\
\mathbf{A} / \mathbf{P}\end{array}$ & $\begin{array}{c}\mathbf{2 5 : 7 5} \\
\mathbf{A} / \mathbf{P}\end{array}$ & $\begin{array}{c}\mathbf{1 0 0} \\
\mathbf{P}\end{array}$ \\
\cline { 2 - 6 } $\mathbf{g}$ & $\mathbf{g}$ & $\mathbf{g}$ & $\mathbf{g}$ & $\mathbf{g}$ \\
\hline $\begin{array}{c}\text { Ruiba } \\
\text { rbo }\end{array}$ & 200 & 200 & 200 & 200 & 200 \\
\hline $\begin{array}{c}\text { Azúca } \\
\mathbf{r}\end{array}$ & $\begin{array}{c}252, \\
6\end{array}$ & 189 & 126 & 63 & $*$ \\
\hline $\begin{array}{c}\text { Panel } \\
\mathbf{a}\end{array}$ & $\star$ & 66.5 & $\begin{array}{c}132 . \\
3\end{array}$ & $\begin{array}{c}198.4 \\
5\end{array}$ & $\begin{array}{c}264 . \\
6\end{array}$ \\
\hline $\begin{array}{c}\text { Pectin } \\
\mathbf{a}\end{array}$ & 1.73 & 1.73 & 1.73 & 1.73 & 1.73 \\
\hline $\begin{array}{c}\text { Benzo } \\
\text { ato de } \\
\text { sodio }\end{array}$ & 0.2 & 0.2 & 0.2 & 0.2 & 0.2 \\
\hline
\end{tabular}

$\mathrm{A}=$ Azúcar $-\mathrm{P}=$ Panela. Fuente: Autores.

Se inició con la selección del peciolo del ruibarbo, troceándolo manualmente. Se procedió a realizar un escaldado durante 5 minutos a una temperatura de $80^{\circ} \mathrm{C}$ (Marquina, V., et al., 2008). Posteriormente se calentó el peciolo troceado, antimicrobianos, pectina y azúcar en una marmita de doble fondo, agitando constantemente hasta alcanzar la ebullición de la mezcla. Al momento de alcanzar una concentración de 65 grados Brix $\left({ }^{\circ} \mathrm{Bx}\right)$. Se envasó la mermelada elaborada a temperatura entre $85^{\circ} \mathrm{C}$ y $90^{\circ} \mathrm{C}$ en frascos de vidrio con la finalidad de obtener el vacío adecuado. La mermelada envasada se pasteurizó en autoclave, almacenándose el producto terminado a temperatura ambiente (López, M., et al., 2011).

La mermelada fue caracterizada a través de análisis fisicoquímicos con dos repeticiones: contenido de sólidos en ${ }^{\circ} \mathrm{Bx}$, se determinó utilizando un refractómetro Abbe; pH por método potencio-métrico, (AOAC, 2000).

Para los análisis microbiológicos realizados a la mermelada se aplicaron las establecidas por la Norma Técnica Colombiana (NTC 285): Mesófilos Aerobios, Coliformes totales, Coliformes fecales, mohos y levaduras.

\section{Análisis Estadístico}

Los resultados correspondientes a tres ensayos independientes se expresaron como el promedio \pm el error estándar de la media (ESM) y se analizaron mediante pruebas $t$ y análisis de varianza de una vía 
@ (2IMENTECH CIENCIA Y TECNOLOGÍA ALIMENTARIA ISSN 1692-7125. Volumen 14, No. 2, p. 33- 41, año 2016 Facultad de Ingenierías y Arquitectura Universidad de Pamplona

(ANOVA), seguido de prueba de Dunnet o Tukey post hoc para comparaciones múltiples. Valores de $\mathrm{P}<0,05$ fueron considerados significativos. Para la organización de los datos se empleó la hoja de cálculo MS Excel 2010, y para los análisis estadísticos el paquete GraphPad Prism V5.00 para Windows.

\section{RESULTADOS}

La Tabla 2 presenta la caracterización fisicoquímica del peciolo de ruibarbo ( $R$. Rhabarbarum).

Tabla 2. Composición fisicoquímica del peciolo del ruibarbo.

\begin{tabular}{|c|c|}
\hline Componente & $\begin{array}{c}\text { Resultados } \\
\text { Obtenidos }\end{array}$ \\
\hline Proteínas & $0.86 \%$ \\
\hline Fibra total & $1.48 \%$ \\
\hline Humedad & $92.27 \%$ \\
\hline Grasas & $0.21 \%$ \\
\hline Carbohidratos & $4.13 \%$ \\
\hline Calcio & $90 \mathrm{mg}$ \\
\hline Potasio & $880 \mathrm{mg}$ \\
\hline Hierro & $0.20 \mathrm{ppm}$ \\
\hline pH & $3.42 \pm 0.50$ \\
\hline
\end{tabular}

Fuente: Autores.

En la tabla 3 muestra la composición fisicoquímica de las cinco (5) formulaciones de mermeladas, en la cual se observa un incremento en el contenido de proteína, calcio, hierro, fibra total, humedad a medida que aumenta la cantidad de panela, puesto que esta presenta un elevado contenido nutricional en comparación con el azúcar por ser un producto natural (Álvarez A., 2000).

Tabla 3. Composición fisicoquímica de las diferentes formulaciones de mermeladas de ruibarbo.

\begin{tabular}{|c|c|c|c|c|c|}
\hline \multirow{3}{*}{$\begin{array}{c}\text { Compon } \\
\text { entes }\end{array}$} & \multicolumn{5}{|c|}{ Resultados } \\
\hline & $100 \mathrm{~A}$ & $\begin{array}{c}75: 25 \\
\text { A/P }\end{array}$ & $\begin{array}{c}50: 50 \\
\text { A/P }\end{array}$ & $\begin{array}{c}25 / 75 \\
\text { A/P }\end{array}$ & $\begin{array}{c}100 \\
P\end{array}$ \\
\hline & $\%$ & $\%$ & $\%$ & $\%$ & $\%$ \\
\hline $\begin{array}{l}\text { Proteín } \\
\text { as }\end{array}$ & $\begin{array}{c}1.66 \\
\pm \\
0.07\end{array}$ & $\begin{array}{c}2.50 \\
\pm \\
0.06\end{array}$ & $\begin{array}{c}3.35 \\
\pm \\
0.05\end{array}$ & $\begin{array}{c}3.61 \\
\pm \\
0.04\end{array}$ & $\begin{array}{c}3.87 \\
\pm \\
0.04\end{array}$ \\
\hline Grasas & $\begin{array}{l}0.4 \pm \\
0.02\end{array}$ & $\begin{array}{c}0.26 \\
\pm \\
0.02\end{array}$ & $\begin{array}{c}0.12 \\
\pm \\
0.03\end{array}$ & $\begin{array}{c}0.13 \\
\pm \\
0.03\end{array}$ & $\begin{array}{c}0.14 \\
\pm \\
0.03\end{array}$ \\
\hline $\begin{array}{c}\text { Carbohid } \\
\text { ratos }\end{array}$ & $\begin{array}{c}73.0 \\
\pm \\
0.19\end{array}$ & $\begin{array}{c}68.9 \\
\pm \\
0.18\end{array}$ & $\begin{array}{c}64.9 \\
\pm \\
0.18\end{array}$ & $\begin{array}{c}61.2 \\
\pm \\
0.23 \\
\end{array}$ & $\begin{array}{c}57.5 \\
\pm \\
0.28\end{array}$ \\
\hline Calcio & $\begin{array}{c}0.03 \\
\pm \\
0.01\end{array}$ & $\begin{array}{c}0.12 \\
\pm \\
0.02\end{array}$ & $\begin{array}{c}0.21 \\
\pm \\
0.03\end{array}$ & $\begin{array}{c}0.31 \\
\pm \\
0.03\end{array}$ & $\begin{array}{c}0.42 \\
\pm \\
0.04\end{array}$ \\
\hline
\end{tabular}


@-LIMENTECH CIENCIA Y TECNOLOGÍA ALIMENTARIA ISSN 1692-7125. Volumen 14, No. 2, p. 33- 41, año 2016 Facultad de Ingenierías y Arquitectura Universidad de Pamplona

\begin{tabular}{|c|c|c|c|c|c|}
\hline Potasio & $\begin{array}{c}0.19 \\
\pm 0.02\end{array}$ & $\begin{array}{c}0.20 \\
\pm\end{array}$ & $\begin{array}{c}0.21 \\
\pm\end{array}$ & $\begin{array}{c}0.22 \\
\pm\end{array}$ & $\begin{array}{c}0.23 \\
\pm\end{array}$ \\
& & 0.02 & 0.02 & 0.01 & 0.01 \\
\hline $\begin{array}{c}\text { Hierro, } \\
\text { ppm }\end{array}$ & $\begin{array}{c}19.3 \\
\pm\end{array}$ & $\begin{array}{c}20.8 \\
\pm\end{array}$ & 22.4 & 24.1 & 25.8 \\
& 0.14 & 0.14 & \pm 0.15 & \pm & \pm \\
& & & 0.13 & 0.12 \\
\hline Fibra & 0.16 & 0.32 & 0.48 & 0.58 & 0.69 \\
total & \pm & \pm & \pm & \pm & \pm \\
& 0.02 & 0.03 & 0.05 & 0.05 & 0.06 \\
\hline Humed & 23.9 & 27.1 & 30.4 & 33.5 & 36.6 \\
ad & \pm & \pm & \pm & \pm & \pm \\
& 0.26 & 0.24 & 0.23 & 0.23 & 0.24 \\
\hline
\end{tabular}

Fuente: Autores.

En la tabla 4 se puede observar que existen diferencias significativas entre las variables de color con las diversas formulaciones.

Tabla 4. Variables colorimétricas en las diferentes formulaciones de mermelada de ruibarbo

\begin{tabular}{|c|c|c|c|}
\hline \multirow[t]{2}{*}{ Formulaciones } & \multicolumn{3}{|c|}{ COLOR } \\
\hline & $L^{*}$ & $a^{*}$ & $\mathbf{b}^{*}$ \\
\hline $100 \mathrm{~A}$ & $\begin{array}{c}30.7^{\mathrm{a}} \pm \\
0.00\end{array}$ & $\begin{array}{c}5.42^{\mathrm{a}} \pm \\
0.01\end{array}$ & $\begin{array}{r}25.8^{a} \\
\pm 0.10 \\
\end{array}$ \\
\hline $75: 25 \mathrm{~A} / \mathrm{P}$ & $\begin{array}{c}23.0^{\mathrm{b}} \pm \\
0.01\end{array}$ & $\begin{array}{c}9.78^{b} \pm \\
0.02\end{array}$ & $\begin{array}{r}23.3^{b} \\
\pm 0.03\end{array}$ \\
\hline $50: 50 \mathrm{~A} / \mathrm{P}$ & $\begin{array}{c}16.0^{\mathrm{c}} \pm \\
0.02\end{array}$ & $\begin{array}{c}8.55^{c} \pm \\
0.02\end{array}$ & $\begin{array}{r}17.2^{c} \\
\pm 0.01 \\
\end{array}$ \\
\hline $25: 75 \mathrm{~A} / \mathrm{P}$ & $\begin{array}{c}20.6^{\mathrm{d}} \pm \\
0.11\end{array}$ & $\begin{array}{l}10.56^{d} \\
\pm 0.02 \\
\end{array}$ & $\begin{array}{l}22.7^{d} \\
\pm 0.57\end{array}$ \\
\hline $100 P$ & $\begin{array}{c}21.1^{\mathrm{e}} \pm \\
0.00\end{array}$ & $\begin{array}{l}11.38^{e} \\
\pm 0.00\end{array}$ & $\begin{array}{c}24.1^{\mathrm{e}} \\
\pm 0.05\end{array}$ \\
\hline
\end{tabular}

Medias seguidas por diferentes letras indican diferencias significativas $(P<0.05)$.

Fuente: Autores.
En la tabla 5 se observa los sólidos solubles y el $\mathrm{pH}$ de las diversas formulaciones destacando que dicho porcentaje cumplen con los requisitos fisicoquímicos para las mermeladas y jaleas de frutas establecido por la NTC 285.

Tabla 5. Contenido de sólidos solubles y pH de las diferentes formulaciones de mermelada de ruibarbo.

\begin{tabular}{|c|c|c|}
\hline Formulaciones & ${ }^{\circ}$ Brix & $\mathrm{pH}$ \\
\hline $100 \mathrm{~A}$ & $\begin{array}{c}67.33^{\mathrm{a}} \pm \\
0.28\end{array}$ & $\begin{array}{c}3.38^{\mathrm{a}} \pm \\
0.02\end{array}$ \\
\hline 75:25 A/P & $\begin{array}{c}62.66^{\mathrm{b}} \pm \\
0.28\end{array}$ & $\begin{array}{c}3.48^{\mathrm{b}} \pm \\
0.01\end{array}$ \\
\hline 50:50 A/P & $\begin{array}{c}61.50^{c} \pm \\
0.50\end{array}$ & $\begin{array}{c}3.49^{b} \pm \\
0.01\end{array}$ \\
\hline 25:75 A/P & $\begin{array}{c}65.00^{d} \pm \\
0.50\end{array}$ & $\begin{array}{c}3.43^{c} \pm \\
0.01\end{array}$ \\
\hline $100 \mathrm{P}$ & $\begin{array}{c}66.50^{\mathrm{e}} \pm \\
0.50\end{array}$ & $\begin{array}{c}3.36^{\mathrm{a}} \pm \\
0.01\end{array}$ \\
\hline
\end{tabular}

Medias seguidas por diferentes letras en indican diferencias significativas $(P<0.05)$

Fuente: Autores.

El análisis microbiológico del producto terminado, se realizó según los criterios microbiológicos de la NTC 285, detallados a continuación en la tabla 6 , en la cual todas las formulaciones presentaron valores aceptados por la normativa, lo que refleja las buenas prácticas de manufactura y correcto almacenamiento del producto terminado. 
@-LIMENTECH CIENCIA Y TECNOLOGÍA ALIMENTARIA ISSN 1692-7125. Volumen 14, No. 2, p. 33- 41, año 2016 Facultad de Ingenierías y Arquitectura Universidad de Pamplona

Tabla 6. Análisis microbiológico de los productos terminados

\begin{tabular}{|c|c|c|c|c|}
\hline $\begin{array}{l}\text { Formula } \\
\text { ciones }\end{array}$ & $\begin{array}{c}\text { Meso } \\
\text { filos } \\
\text { Aero } \\
\text { bios } \\
\text { UFC/ } \\
\text { g }\end{array}$ & $\begin{array}{c}\text { Colifo } \\
\text { rmes } \\
\text { totale } \\
\text { s } \\
\text { UFC/g }\end{array}$ & $\begin{array}{c}\text { Colifo } \\
\text { rmes } \\
\text { fecale } \\
\text { s } \\
\text { UFC/g }\end{array}$ & $\begin{array}{c}\text { Moho } \\
\text { s y } \\
\text { levad } \\
\text { uras } \\
\text { UFC/ } \\
\text { g }\end{array}$ \\
\hline $100 \mathrm{~A}$ & $<10$ & $<10$ & $<10$ & 30 \\
\hline $\begin{array}{l}75: 25 \\
\text { A/P }\end{array}$ & $<10$ & $<10$ & $<10$ & $<10$ \\
\hline $\begin{array}{l}50: 50 \\
\text { A/P }\end{array}$ & $<10$ & $<10$ & $<10$ & $<10$ \\
\hline $\begin{array}{l}25: 75 \\
A / P\end{array}$ & $<10$ & $<10$ & $<10$ & $<10$ \\
\hline $100 \mathrm{P}$ & $<10$ & $<10$ & $<10$ & $<10$ \\
\hline $\begin{array}{l}\text { Valor } \\
\text { Referenc } \\
\text { ia }\end{array}$ & $\begin{array}{l}<300 \\
\text { ufc/g }\end{array}$ & $\begin{array}{l}<10 \\
\text { ufc/g }\end{array}$ & $\begin{array}{l}<10 \\
\text { ufc/g }\end{array}$ & $\begin{array}{l}<50 \\
\text { ufc/g }\end{array}$ \\
\hline
\end{tabular}

UFC $=$ Unidades Formadora de Colonias.

Fuente: Autores.

Los resultados obtenidos para la caracterización fisicoquímica son similares a los incluidos por la base de datos nacional de nutrientes USDA, 2016 (United States Department of Agriculture).

\section{CONCLUSIÓN}

Se puede decir que el ruibarbo es una fuente económica y nutritiva para el desarrollo de mermeladas e igualmente la presencia de panela en las formulaciones evidencio un
Desde el punto de vista tecnológico, el pH es un parámetro fisicoquímico importante en el control del desarrollo de poblaciones de microorganismos, responsable de la actividad de sistemas enzimáticos, en el proceso de clarificación de jugos y bebidas, en la estabilidad de los mismos y de otros productos elaborados a partir de frutas; así como en la producción de jalea y mermelada cuya firmeza, color y flavor están determinados por la concentración de iones hidrógenos (Carvajal, et al., 2012; MooHuchin, et al., 2015; Ramírez, R., et al., 2012).

La utilización de la panela produce un cambio en el color característico de la mermelada, prevaleciendo el color característico de la panela; Martínez (2014) afirma que el color en la panela es un atributo variable que depende factores propios del cultivo como la variedad de la caña, tipo de suelo y de factores de producción como el tiempo y la temperatura de cocción de los jugos, métodos e insumos utilizados durante la clarificación (Martínez A., 2014). aumento en el contenido de proteína, calcio, hierro, fibra total y humedad a medida que aumenta la concentración de la panela adicionada. 


\section{AGRADECIMIENTOS}

Los autores agradecen al SENA, Centro de Comercio y Servicio de la Regional Bolívar y a la Universidad de Cartagena, por su apoyo en el desarrollo de este trabajo.

\section{REFERENCIAS}

Álvarez A. (2000). Panela en estados unidos. Corporación Colombia internacional. Bogotá (Colombia). 2000. Publicación $\mathrm{N}^{\circ} 26$.

AOAC, 2000. Official Methods of Analysis. Section 932.12. and 981.12. 14th. Ed. Association of Oficial Analytical Chemist. Washington D.C. USA.

Carvajal, LM, El Hadi Yahia, C., Cartagena, R., Peláez, C., Gaviria, C.A., Rojano, B.A. (2012). Capacidad antioxidante de dos variedades de Fragaria $x$ ananassa (Weston) Duchesne (fresa) sometidas a variaciones en la nutrición vegetal. Revista Cubana de Plantas Medicinales. 17(1)37-53.

Hisashi, M., Toshio, M., Iwao, T., Ji-Young, P., Shoichi, H., Masayuki, Y. (2001). Antioxidant Constituents from Rhubarb: Structural Requirements of Stilbenes for the Activity and Structures of Two New Anthraquinone Glucosides. Bioorganic \&

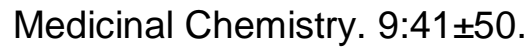

Huber, T., Graupner, N., Müssig, J. (2009).

As tough as it is delicious? A mechanical and structural analysis of red rhubarb (Rheum rhabarbarum). J Mater Sci. 44:4195-4199.

López, M., Mercado, J., Martínez, G., Magaña, J.L. (2011). Formulación de una mermelada a partir de pulpa y cáscara de tunas (Opuntia spp.) elaborada a nivel planta piloto. Acta Universitaria. 21: 31 36.

Marquina, V., Araujo, L., Ruíz, J., RodríguezMalaver, A., Vit, P. (2008). Composición química y capacidad antioxidante en fruta, pulpa y mermelada de guayaba (Psidium guajava L.). Archivos Latinoamericanos de Nutrición. 58: 98-102.

Martínez A., (2014). Hay que desmitificar la creencia de que el color determina la calidad de la panela. Periódico el Espectador.

http://www.elespectador.com/noticias/pub lirreportaje/hay-desmitificar-creencia-deel-color-determina-calidad-articulo525295. Accesado: 17 febrero 2016.

McDougall, G., Dobson, P., Jordan-Mahy, N., (2010) Effect of different cooking regimes 
@. LIMENTECH CIENCIA Y TECNOLOGÍA ALIMENTARIA ISSN 1692-7125. Volumen 14, No. 2, p. 33- 41, año 2016 Facultad de Ingenierías y Arquitectura Universidad de Pamplona

on rhubarb polyphenols. Food Chemistry. ; 119: 758-764.

Moo-Huchin, VM., Moo-Huchin, MI., EstradaLeón, RJ., Cuevas-Glory, L. EstradaMota, IA., Ortiz-Vázquez, E., BetancurAncona, D., Sauri-Duch, E. (2015). Antioxidant compounds, antioxidant activity and phenolic content in peel from three tropical fruits from Yucatan, Mexico. Food Chemistry. 166: 17-22.

Morillas-Ruiz, JM. y Delgado-Alarcón, J. M., (2012). Análisis nutricional de alimentos vegetales con diferentes orígenes: Evaluación de capacidad antioxidante y compuestos fenólicos totales. Nutr. clín. diet. hosp. 32(2):8-20.

Öztürk, M., Aydoğmuş-Öztürk, F., Duru, M.E., Topçu, G. (2007). Antioxidant activity of stem and root extracts of Rhubarb (Rheum ribes): an edible medicinal plant. Food Chem; 103, 623630.

Paula, J., Martins, A., (2000). Acción antibacteriana de extractos hidroalcohólicos de Rubus urticaefolius. Rev Cubana Plant Med. 5(1): 26-29.

Ramírez, R., Arenas, L., Acosta, K., Yamarte, M., Sandoval, L., (2012). Efecto del escaldado sobre la calidad nutricional de pulpa de Guanábana (Annona muricata
L.). Revista Iberoamericana de Tecnología Postcosecha. 13(1): 48-57.

Santamaría, F., Díaz, R., Sauri, E., Gil, F., Santamaría, J., Larque, A. (2010). Características de calidad de frutos de papaya maradol en la madurez de consumo. Agric. Tecn. Mex. 35(3):347353.

Torrenegra, M., Granados, C., Osorio, M., León, G. (2015). Method comparison of hydrodistillation microwave radiationassisted (MWHD) front hydrodistillation (HD) in the extraction of essential oil of Minthostachys mollis. Inf. Tecnol. 26(1): 117-122.

USDA. BasicReport: 09307, Rhubarb, raw. Agricultural Research Service. United States Department of Agriculture.http://ndb.nal.usda.gov/ndb/fo ods/show/2377?format=Abridged\&reportf $\mathrm{mt}=$ pdf\&pdfQvs=\%7B\%7D. Accesado: 17 febrero 2016.

Yáñez, C. y Santafé, G., (2012). Evaluación de la actividad antioxidante del aceite esencial foliar de Calycolpus moritzianus y Minthostachys mollis de Norte de Santander. Bistua: Revista de la Facultad de Ciencias Básicas. Volumen 10 (1),1223. 